\title{
Factores de Vulnerabilidad y de Protección del Impacto Emocional en Mujeres Adultas Víctimas de Agresiones Sexuales
}

\section{Risk and Protective Factors in the Emotional Impact of Adult Female Victims of Sexual Aggression}

\author{
Belén Sarasua \\ Irene Zubizarreta \\ Centro de Tratamiento Psicológico para Víctimas de Violencia de Género. Programa de la Diputación Foral de Álava, \\ España \\ Paz de Corral \\ Enrique Echeburúa \\ Universidad del País Vasco UPV/EHU, Cibersam, España.
}

(Rec: 13 de junio de 2012 / Acep: 06 de julio de 2012)

\begin{abstract}
Resumen
El objetivo de este estudio fue describir las reacciones psicopatológicas de las víctimas según las circunstancias de la agresión sexual, la historia previa y el apoyo familiar/social. La muestra constó de 269 mujeres adultas víctimas de una agresión sexual en la infancia o en la vida adulta. Se evaluaron las reacciones postraumáticas, la sintomatología ansioso-depresiva, la autoestima, las conductas sexuales y el funcionamiento cotidiano. Los resultados mostraron una alta prevalencia de malestar emocional $(63,6 \%)$, de baja autoestima $(59,7 \%)$, de TEPT (44,5\%), de sentimientos de culpa $(48,3 \%)$ y de evitación sexual $(38,9 \%)$, así como problemas de adaptación. La gravedad de la sintomatología estaba relacionada con las circunstancias de la agresión sexual, tales como la penetración vaginal/anal o las heridas provocadas, la historia de victimización, los sucesos estresantes recientes y la falta de apoyo socio-familiar. Sin embargo, las conductas de evitación sexual no estaban relacionadas con circunstancias específicas de la agresión sexual.

Palabras clave: agresión sexual, mujeres adultas víctimas, impacto psicológico, factores de vulnerabilidad y de protección.
\end{abstract}

\begin{abstract}
The aim of this paper was to describe the psychopathological reactions of the victims according to the circumstances surrounding sexual aggression, the previous clinical record, and the role of family/social support. The sample consisted of 269 adult female victims who suffered any kind of sexual aggression either in childhood or in adult life. Participants were assessed with the PTSD Severity Scale, the STAI, the BDI, the Self-Esteem Scale and the Misadjustment Scale. The results showed a high prevalence of emotional trouble (63.6\%), low self-esteem (59.7\%), PTSD (44.5), guilt feelings (48.3\%), sexual avoidance (38.9\%), as well as a poor adaptation to daily life activities. The severity of emotional problems was related to the circumstances of sexual aggression, such as vaginal/anal penetration or physical injuries, the history of victimization, the recent stressful life events and the lack of family/social support. However sexual avoidance behaviors were not associated with circumstances of sexual aggression.

Keywords: sexual aggression, adult female victims, psychological impact, risk and protective factors.
\end{abstract}

Agradecimientos: Este estudio ha sido apoyado por el Grupo Consolidado de Investigación en Psicología Clínica del Gobierno Vasco y por la UFI 11/04 de la Universidad del País Vasco (UPV/EHU).

Correspondencia: Enrique Echeburúa. Facultad de Psicología. Avda. de Tolosa, 70.20018 San Sebastián. E-mail: enrique.echeburua@ehu.es 


\section{Introducción}

Si bien cualquier persona puede ser víctima de una agresión sexual, son las mujeres quienes padecen en mayor medida esta conducta violenta. De hecho, cerca de un $15 \%$ de la población femenina sufre alguna agresión sexual a lo largo de su vida (Campbell y Wasco, 2005; Vickerman y Margolin, 2009). Asimismo, según un reciente meta-análisis de Pereda, Guilera, Forns y Gómez-Benito (2009), la tasa de prevalencia de algún tipo de abuso sexual en menores en la población general de diversos países es de un $7.4 \%$ en el caso de los niños y de un $19.2 \%$ en el de las niñas.

La victimización -el hecho de ser víctima de un suceso violento- puede causar unas repercusiones psicológicas muy negativas en la estabilidad emocional de las personas afectadas, especialmente en el caso de las mujeres agredidas sexualmente en la vida adulta o en la infancia (Cortés, Cantón-Cortés y Cantón, 2011; Echeburúa y Guerricaechevarría, 2011; PicóAlfonso, Echeburúa y Martínez, 2008). Así, el desarrollo del trastorno de estrés postraumático (TEPT) como consecuencia de cualquier conducta violenta lo experimenta el $25 \%$ de todas las víctimas, pero este porcentaje puede ascender hasta el $50-60 \%$ en el caso de las mujeres agredidas sexualmente (Echeburúa, Corral, Amor, Zubizarreta y Sarasua, 1997; Foa y Rothbaum, 1998).

Las agresiones sexuales son sucesos traumáticos que pueden producir consecuencias psicológicas intensas a corto y largo plazo en las víctimas. La victimización sexual es uno de los delitos más violentos por naturaleza. La humillación y el miedo a sufrir daños físicos y psíquicos e incluso a ser asesinadas produce una reacción de indefensión y pérdida de control que afecta al equilibrio emocional e interfiere en el desarrollo de una vida normal (Echeburúa, Corral, Sarasua y Zubizarreta, 1996; Resnick et al., 2007).

De hecho, más del $50 \%$ de las víctimas padecen un trastorno de estrés postraumático (TEPT) y un malestar emocional clínicamente significativo (Echeburúa, Corral, Zubizarreta y Sarasua, 1997; Foa, Zoellner y Feeny, 2006), además de conductas de evitación sexual, incluso en el caso de que hayan sufrido la agresión meses o años atrás (Sarasua, Zubizarreta, Corral y Echeburúa, 2012, en prensa). Así, las víctimas de abuso sexual infantil pueden experimentar en la vida adulta una tasa de trastornos mentales 2.4 veces mayor que las personas no expuestas a este suceso traumático (Fergusson, Boden y Horwood, 2008; Resick, Williams, Suvak, Monson y Gradus, 2012).

Las reacciones emocionales negativas (TEPT, síntomas ansioso-depresivos, sentimientos de culpa, inadaptación a la vida cotidiana, evitación sexual) suelen ser más graves y duraderas cuando el suceso sufrido es muy intenso o cuando la víctima es vulnerable psicológicamente y no cuenta con un apoyo familiar y social adecuado. La intensidad de los síntomas inmediatamente tras la agresión es un predictor del funcionamiento de las víctimas a largo plazo (Echeburúa, 2010; Resnick et al., 2007).

En concreto, el impacto psicológico de las agresiones sexuales va a depender de la frecuencia, de la duración, de la gravedad del abuso y de la relación con el agresor, pero también de la fase del desarrollo psicológico de la víctima en la que tuvo lugar el suceso (Briere y Elliott, 2003; Echeburúa, Sarasua, Zubizarreta y Corral, 2012, en prensa). Además, las circunstancias de la agresión sexual, la historia de victimización, la denuncia, la reacción judicial y el apoyo familiar y social existente pueden agravar o amortiguar, según los casos, las reacciones emocionales de las víctimas (Echeburúa y Corral, 2007; Echeburúa, Corral y Amor, 2002).

Sin embargo, los estudios existentes hasta la fecha en relación con los factores psicosociales de vulnerabilidad y de protección de la víctima son muy generales, están muy centrados en el TEPT como respuesta psicopatológica y no permiten obtener resultados concluyentes sobre el papel agravante o amortiguador de las variables psicosociales implicadas específicamente en la respuesta psicológica global a una agresión sexual. Por ello, el objetivo de este estudio es describir las reacciones psicopatológicas de las víctimas (TEPT, malestar emocional, sentimientos de culpa, déficits de autoestima y evitación sexual) y ponerlas en relación con las circunstancias de la agresión, la presentación de una denuncia, la historia de victimización y la presencia o ausencia de apoyo familiar y social. Todo ello permitirá anticipar la reacción emocional específica de una víctima y establecer, por tanto, un tratamiento diseñado más a la medida, con la finalidad de conseguir mejores resultados y reducir la tasa de abandonos prematuros de la terapia.

\section{Método}

\section{Participantes}

La muestra total de este estudio consta de 269 mujeres que han sufrido una agresión sexual y que han acudido en busca de un tratamiento psicológico al Centro de Asistencia Psicológica para Víctimas de Violencia de Género de la Diputación Foral de Álava (País Vasco). En dicho centro se desarrolla un programa de tratamiento específico para víctimas de agresiones sexuales desde 1990 y la atención ofertada es gratuita.

Los criterios de selección han sido los siguientes: a) ser mujer mayor de 18 años y haber sido víctima de una agresión sexual, bien en la vida adulta, bien en la infancia $\mathrm{o}$ adolescencia; b) no haber sufrido la agresión por parte de su pareja ni haber estado expuesta a un acoso sexual en el trabajo; c) no sufrir actualmente un proceso de victimización ni un trastorno mental grave; $y \mathrm{~d}$ ) acceder voluntariamente a la participación en el estudio tras haber sido ampliamente informada, con la firma de una hoja de consentimiento. 
La muestra preseleccionada abarcó a las 289 mujeres que habían acudido al programa. Sin embargo, 11 (4\%) fueron excluidas por haber sufrido una agresión sexual por parte de su pareja, $6(2 \%)$ por haber estado expuestas a un acoso sexual en el trabajo y $3(1 \%)$ por padecer un trastorno mental grave (depresión mayor). La muestra seleccionada finalmente fue, por tanto, de 269 mujeres.

Respecto a las características demográficas de la muestra total, las víctimas eran mujeres jóvenes, con una edad media de 27.6 años ( $D T=8.9$; rango: 18-58), no tenían pareja en una mayoría de los casos $(n=165 ; 61.3 \%)$, eran de nacionalidad española $(n=226 ; 84 \%)$ y pertenecían a un nivel socioeconómico medio $(n=151 ; 56.1 \%)$.

\section{Diseño}

En este estudio se ha utilizado un diseño ex post facto de tipo retrospectivo, en el que se cuenta con dos variables (sí o no) en función de cada uno de los cinco objetivos de la investigación (trastorno de estrés postraumático, malestar emocional, sentimiento de culpa, déficit de autoestima y evitación de la conducta sexual).

\section{Instrumentos}

\section{a) Variables sociodemográficas, circunstancias del proceso de victimización y apoyo familiar y social}

La Entrevista Semiestructurada para Victimas de Agresiones Sexuales (Echeburúa, Corral, Zubizarreta y Sarasua, 1995) es un instrumento que evalúa la historia de victimización sexual, la percepción de amenaza a la vida y las consecuencias jurídico-penales. Asimismo permite identificar la reacción del entorno familiar, los recursos de apoyo existentes, la victimización secundaria y el sentimiento de culpa. Por último, se reflejan las características sociodemográficas, los antecedentes psicopatológicos, la existencia de sucesos estresantes en el último año y el estado de salud de las víctimas.

\section{b) Variables psicopatológicas}

La Escala de Gravedad de Síntomas del Trastorno de Estrés Postraumático (EGS) (Echeburúa et al., 1997) es una escala heteroaplicada que evalúa la gravedad e intensidad de los síntomas de este trastorno según los criterios diagnósticos del DSM-IV-TR (APA, 2000) en víctimas de diferentes sucesos traumáticos. Esta escala está estructurada en un formato de tipo Likert, de 0 a 3 , según la frecuencia e intensidad de los síntomas y consta de 17 ítems que corresponden a los criterios diagnósticos (5 hacen referencia a los síntomas de reexperimentación; 7 a los de evitación; y 5 a los de activación). El rango oscila de 0 a 51 en la escala global; de 0 a 15 en la subescala de reexperimentación; de 0 a 21 en la de evitación; y de 0 a 15 en la de activación.
Es una escala con una eficacia diagnóstica muy alta $(95.45 \%)$ si se establece un punto de corte global de $15 \mathrm{y}$ unos puntos de corte parciales de 5 (con 2 puntos, al menos, en un síntoma), 6 (con 2 puntos, al menos, en tres síntomas) y 4 (con dos puntos, al menos, en dos síntomas) en las subescalas de reexperimentación, evitación y activación, respectivamente. Las propiedades psicométricas son muy satisfactorias (Echeburúa et al., 1997).

La Entrevista Semiestructurada para Víctimas de Agresiones Sexuales (Echeburúa, Corral, Zubizarreta y Sarasua, 1995) incluye una pregunta directa en relación con el sentimiento de culpa que resulta de la atribución interna de lo que "podía haber hecho y no hizo para evitar la agresión" con dos opciones de respuesta (afirmativa o negativa).

El Inventario de Ansiedad-Estado (STAI) (Spielberger, Gorsuch y Lushene, 1970) (versión española de TEA, 1982) es un autoinforme que consta de 20 ítems relacionados con la ansiedad-rasgo y de otros 20 relacionados con la ansiedad-estado. El rango de puntuaciones oscila de 0 a 60 en cada escala. El punto de corte elegido para la población femenina en el STAI-Estado es de 31 (correspondiente al percentil 75). La fiabilidad test-retest es de 0.81 en la escala de ansiedad-rasgo, bastante más alta, como es lógico, que en la de ansiedad-estado $(r=.40)$. La consistencia interna varía de .83 a .92 . Por otra parte, la validez convergente con otras medidas de ansiedad oscila entre .58 y .79 .

El Inventario de Depresión de Beck (BDI) (Beck, Rush, Shaw y Emery, 1979) (versión española de Vázquez y Sanz, 1997) es un autoinforme de 21 ítems (rango: 0-63 puntos) que mide la intensidad de los síntomas depresivos y da más importancia a los componentes cognitivos de la depresión que a los conductuales y somáticos. El punto de corte más utilizado para discriminar entre población sana y aquejada de sintomatología depresiva es de 18 (Beck, Brown y Steer, 1996). El coeficiente de fiabilidad por el método de las dos mitades es de .93. Desde la perspectiva de la validez convergente, la correlación con la evaluación clínica de la depresión oscila entre .62 y .66.

La Escala de Autoestima (EAE) (Rosenberg, 1965) tiene por objetivo evaluar el grado de satisfacción que tiene una persona consigo misma. Este autoinforme consta de 10 ítems generales que puntúan de 1 a 4 en una escala de tipo Likert (rango: 10-40 puntos). El punto de corte en la población adulta es de 29 , con una puntuación tanto mayor cuanto mayor es la autoestima. La fiabilidad test-retest es de .85 y el coeficiente alfa de consistencia interna es de .92. La validez convergente y la validez discriminante son asimismo satisfactorias. La versión española de esta escala se puede encontrar en Echeburúa y Corral (1998).

La Escala de Inadaptación (IG) (Echeburúa, Corral y Fernández-Montalvo, 2000) es un autoinforme que consta de 6 ítems (rango: 0-30) y mide el grado en que la situación de maltrato afecta a diferentes áreas de la vida cotidiana 
(trabajo, vida social, tiempo libre, relación de pareja o posibilidad de tenerla y relación familiar), así como también a nivel global. El punto de corte establecido es de 12 en la escala total y de 2 en cada uno de los ítems, con una puntuación tanto mayor cuanto mayor es la inadaptación. En esta investigación se ha utilizado el ítem que refleja el grado de inadaptación global a la vida cotidiana (rango: 0-5 puntos). Las propiedades psicométricas de fiabilidad y validez han resultado satisfactorias y están descritas en Echeburúa et al. (2000).

\section{Procedimiento}

Las víctimas participantes en esta investigación fueron evaluadas en el momento de establecer contacto con el programa de Asistencia Psicológica para Víctimas de Violencia de Género de la Diputación Foral de Álava (País Vasco). El protocolo de evaluación fue aplicado en dos sesiones. En la primera se obtuvieron los datos relacionados con las características biográficas y las circunstancias del proceso de victimización sexual y del apoyo sociofamiliar. Y en la segunda se evaluaron los síntomas psicopatológicos. Asimismo, las víctimas firmaron la hoja de consentimiento informado.

La evaluación se llevó a cabo por un equipo de psicólogas clínicas con formación en violencia de género. Además, se realizó una supervisión por las directoras del programa, que cuentan con una dilatada experiencia en el tratamiento de las víctimas de agresiones sexuales.

La fase de evaluación formaba parte de una intervención clínica y, por ello, la terapeuta evaluadora realizaba, posteriormente, el programa de tratamiento.

\section{Análisis de datos}

Los análisis estadísticos se realizaron con el programa SPSS y se utilizó la versión 15.0 para Windows. Para describir las características de la muestra (las variables biográficas e historia de victimización, así como las variables psicopatológicas) se llevó a cabo un análisis de carácter descriptivo (medias, desviaciones típicas y porcentajes). Y para analizar la relación de las variables psicopatológicas con el resto de las variables objeto de estudio (las características de la agresión sexual, así como las variables relacionadas con la víctima y el apoyo recibido) se utilizó un análisis de frecuencias y se recurrió como pruebas de contraste a $t$ y a $X^{2}$, según se tratase de variables cuantitativas o categóricas, respectivamente.

\section{Resultados}

En este apartado se incluyen las características de la agresión sexual y las variables psicopatológicas de la muestra, así como los resultados referidos a la relación entre ambos grupos de variables.

\section{Características de la muestra}

A continuación se describen las características del proceso de victimización y las variables más significativas relacionadas con la víctima y con el apoyo familiar y social de las personas seleccionadas en el estudio $(n=269)$.

Respecto a las circunstancias de la agresión sexual, casi la mitad de las víctimas sufrieron una penetración vaginal o anal $(n=124 ; 46.1 \%)$ en la infancia $(n=131 ; 48.7 \%)$ y algo más de la mitad $(n=138 ; 51.3 \%)$ en la vida adulta, perpetrada, en la mayoría de los casos, por un conocido ( $n=192$; $71.4 \%)$. La mitad sufrió la agresión de forma repetida $(n=133 ; 49.4 \%)$, la mayoría $\sin$ lesiones $(n=218 ; 81 \%)$ y $\sin$ presencia de armas como medio coactivo $(n=239 ; 88.8 \%)$, pero casi una de cada tres víctimas se sintió en peligro de muerte $(n=82 ; 30.5 \%)$.

En cuanto a las variables más significativas relacionadas con la víctima y con el apoyo familiar y social, la mayoría no tenía otra historia de victimización $(n=199 ; 74 \%)$ ni problemas de consumo abusivo de alcohol u otras drogas ( $n=246 ; 91.4 \%$ ), pero casi la mitad de las víctimas estuvieron expuestas a sucesos estresantes en el último año $(n=110$; $40.9 \%)$. Por otro lado, la mayor parte de ellas disponían de una red de apoyo social $(n=182 ; 67.7 \%)$.

Las víctimas revelaron mayoritariamente el suceso a la familia $(n=222 ; 82.5 \%)$, se sintieron creídas $(n=178$; $80.2 \%)$ y más de la mitad recibieron un apoyo eficaz ( $n=131$; $59 \%$ ), pero la denuncia solo se efectuó en la mitad de los casos ( $n=133 ; 49.4 \%)$. Además, en casi un tercio de ellos se produjo una victimización secundaria $(n=37 ; 27.8 \%)$.

La denuncia estuvo relacionada con las características de la agresión sexual. Las víctimas que denunciaron sufrieron con mayor frecuencia una violación (vaginal o anal) $(\mathrm{n}=73 ; 54.9 \%)$, con lesiones físicas $(\mathrm{n}=41 ; 30.8 \%)$ y con la presencia de armas como medio coactivo $(\mathrm{n}=25 ; 18.8 \%)$ en comparación con las que no denunciaron $\left[\mathrm{X}^{2}(1)=8.181\right.$; $\left.\mathrm{p}<.01 ; \mathrm{X}^{2}(1)=24.115 ; \mathrm{p}<.001 ; \mathrm{y} \mathrm{X}^{2}(1)=15.515 ; \mathrm{p}<.001\right]$, respectivamente. Asimismo, las víctimas que denunciaron fueron agredidas con mayor frecuencia en la edad adulta $(\mathrm{n}=116 ; 87.2 \%)$ y por un desconocido $(\mathrm{n}=67 ; 50.4 \%)$ en comparación con las que no denunciaron la agresión sexual $\left[\mathrm{X}^{2}(1)=135.837 ; \mathrm{p}<.001 ; \mathrm{y} \mathrm{X}^{2}(1)=60.919 ; \mathrm{p}<.001\right]$, respectivamente.

A su vez, las víctimas que denunciaron tuvieron con mayor frecuencia una percepción subjetiva de amenaza a la vida $(n=68 ; 51.1 \%)$ en comparación con las que no denunciaron $(n=14 ; 10.3 \%)\left[X^{2}(1)=52.908 ; p<.001\right]$.

Por último, la denuncia estaba asociada con variables de protección y de apoyo. Las víctimas que denunciaron contaron a su familia lo sucedido en mayor proporción $(n=119 ; 89.5 \%)$ que las que no denunciaron $(n=103 ; 75.7 \%)\left[X^{2}(1)=8.802\right.$; $p<.01]$ y tuvieron con mayor frecuencia credibilidad ( $n=114$; $95.8 \%)$, apoyo familiar $(n=96 ; 80.7 \%)$ y social $(n=99 ; 80 \%)$ en comparación con las que no denunciaron $\left[X^{2}(1)=39.369\right.$; 
$p<.001 ; X^{2}(1)=49.763 ; p<.001 ;$ y $\left.X^{2}(1)=5.523 ; p<.05\right]$, respectivamente. A su vez, la denuncia también estuvo relacionada con la petición de ayuda a corto plazo (hasta tres meses después de la agresión). Las víctimas que denunciaron sufrieron la agresión de forma reciente en mayor proporción $(n=82 ; 71.3 \%)$ que las que no denunciaron $(n=10 ; 8.3 \%)$ $\left[X^{2}(1)=98.509 ; p<.001\right]$.

\section{Síntomas psicopatológicos}

Las variables psicopatológicas medidas en la evaluación pretratamiento, que abarcó a 236 víctimas ( 33 de ellas, es decir el 14\%, abandonaron el programa), son el trastorno de estrés postraumático (TEPT), el malestar emocional, considerado éste como una valoración conjunta del nivel de ansiedad, de depresión e inadaptación según los puntos de corte de los instrumentos utilizados ( $\geq 24$ en el STAI-E; $\geq 18$ en el $B D I ; \mathrm{y} \geq 3$ en la $I G$ ), los sentimientos de culpa, la baja autoestima ( $<29$ en la $E A E$, de acuerdo con el punto de corte establecido) y los problemas de evitación sexual.

Las víctimas padecían un trastorno de estrés postraumático en el $44.5 \%$ de los casos evaluados y desde una perspectiva dimensional era grave, con una puntuación global ( $X=20.71 ; D T=10.96)$ claramente por encima del punto de corte establecido. A efectos de comprobar la fiabilidad entre observadores del diagnóstico del TEPT con la $E G S$, se calculó el coeficiente kappa, que fue más bien alto (.91).

Asimismo, las víctimas mostraban un malestar emocional en un $63.6 \%$ de los casos, sentimientos de culpa en un $48.3 \%$ y baja autoestima en un $59.7 \%$ del total de la muestra. A su vez, las víctimas que tenían pareja en el momento de la evaluación psicopatológica $(n=90)$ evitaron mantener relaciones sexuales en el $38.9 \%$ de los casos (tabla 1).

Tabla 1. Frecuencia de las variables psicopatológicas.

\begin{tabular}{lc}
\hline Variables psicopatológicas & $\begin{array}{c}\text { Muestra total } \\
(\mathrm{n}=236) \\
\mathrm{N}(\%)\end{array}$ \\
\hline Trastorno de estrés postraumático & $105(44.5)$ \\
Malestar emocional & $150(63.6)$ \\
Sentimiento de culpa & $114(48.3)$ \\
Déficit de autoestima & $141(59.7)$ \\
Evitación de la conducta sexual $(\mathrm{n}=90)$ & $35(38.9)$ \\
\hline
\end{tabular}

Por último, las víctimas que experimentaron un TEPT estaban peor emocionalmente dado que padecían con mayor frecuencia malestar emocional $(n=90 ; 85.7 \%)$, sentimientos de culpa $(n=74 ; 70.5 \%)$, baja autoestima $(n=87 ; 82.9 \%)$ y problemas sexuales $(n=22 ; 66.7 \%)$, en comparación con las que no presentaron dicho trastorno $\left[X^{2}(1)=40.087\right.$; $\left.p<.001) ; X^{2}(1)=37.236 ; p<.001\right) ; X^{2}(1)=39.268 ; p<.001 ; \mathrm{y}$ $\left.X^{2}(1)=16.917 ; p<.001\right]$, respectivamente.
Resultados de la relación entre las características de la agresión sexual, las variables relacionadas con la víctima y el apoyo recibido con los sintomas psicopatológicos

En este apartado se presentan los resultados referidos a las características de la agresión sexual y a las variables más significativas relacionadas con la víctima y con el apoyo sociofamiliar recibido en relación con los síntomas psicopatológicos: el TEPT, el malestar emocional, los sentimientos de culpa, la baja autoestima y los problemas de evitación sexual.

\section{a) Características de la agresión sexual y variables relacionadas con la víctima y con el apoyo recibido en relación con el TEPT}

Las víctimas que manifestaron un TEPT sufrieron con mayor frecuencia una agresión con penetración vaginal y anal (el 57.1\%), tuvieron lesiones (el 24.8\%) y fueron agredidas bajo la amenaza de un arma (el 12.2\%), en comparación con las que no desarrollaron el TEPT. Asimismo, la exposición a sucesos especialmente estresantes en el último año (el 49.5\%), los problemas de consumo abusivo de alcohol u otras drogas (el 12.4\%) y la falta de apoyo social (el 39.1\%) estuvieron presentes en mayor proporción en las víctimas con TEPT (tabla 2).

\section{b) Características de la agresión sexual y} variables relacionadas con la víctima y con el apoyo recibido en relación con el malestar emocional

Las víctimas con un malestar emocional significativo sufrieron con mayor frecuencia una agresión sexual repetida (el 57.3\%), perpetrada por un conocido (el 77.3\%), así como una victimización secundaria (el 36.2\%), en comparación con las que no lo manifestaron. Asimismo, la exposición a sucesos especialmente estresantes en el último año (el 50\%), los problemas de abuso de alcohol u otras drogas (el 10.7\%) y la falta de apoyo tanto social (el 38\%) como familiar (el $46.5 \%$ ) y de pareja (el $27.8 \%$ ) estuvieron presentes en mayor proporción en las víctimas con malestar emocional (tabla 3).

Además, las víctimas con malestar emocional tardaron más tiempo en ponerse en contacto con el programa $(X=9.3$ años; $D T=10.6$ años) que las que no lo experimentaron $(X=5.9$ años; $D T=8.5$ años $)[t(234)=2.461 ; p<.05]$.

\section{c) Características de la agresión sexual y} variables relacionadas con la víctima y con el apoyo recibido en relación con el sentimiento de culpa

Las víctimas que se sintieron culpables de lo ocurrido sufrieron con mayor frecuencia la agresión sexual durante su infancia (el 59.6\%), perpetrada por un conocido (el 82.5\%), en comparación con las que no se culparon. Asimismo, 
Tabla 2. Relación entre las características de la agresión sexual y las variables relacionadas con la víctima y el apoyo recibido en relación con el trastorno de estrés postraumático (TEPT)

\begin{tabular}{|c|c|c|c|c|}
\hline & $\begin{array}{c}\text { Muestra }(\mathrm{N}=236) \\
\mathrm{N}(\%) \\
\end{array}$ & $\begin{array}{c}\text { Con TEPT }(\mathrm{N}=105) \\
\mathrm{N}(\%) \\
\end{array}$ & $\begin{array}{c}\text { Sin TEPT }(\mathrm{N}=131) \\
\mathrm{N}(\%)\end{array}$ & $\chi^{2}(\mathrm{gl})$ \\
\hline \multicolumn{5}{|l|}{ Tipo de agresión } \\
\hline Con penetración & $108(45.8)$ & $60(57.1)$ & $48(36.6)$ & \\
\hline Sin penetración & $128(54.2)$ & $45(42.8)$ & $83(63.4)$ & $9.870(1)^{* *}$ \\
\hline \multicolumn{5}{|l|}{ Edad del abuso } \\
\hline Infancia & $121(51.3)$ & $54(51,4)$ & $67(51.1)$ & $.002(1)$ n.s. \\
\hline Edad adulta & $115(48.7)$ & $51(48.6)$ & $64(48.9)$ & \\
\hline \multicolumn{5}{|l|}{ Frecuencia } \\
\hline Ocasional & $114(48.3)$ & $52(49.5)$ & $62(47.3)$ & $.113(1)$ n.s. \\
\hline Repetida & $122(51.7)$ & $53(50.5)$ & $69(52.7)$ & \\
\hline \multicolumn{5}{|l|}{ Lesiones } \\
\hline Sí & $41(17.4)$ & $26(24.8)$ & $15(11.4)$ & $7.195(1) * *$ \\
\hline No & $195(82.6)$ & $79(72.2)$ & $116(88.6)$ & \\
\hline \multicolumn{5}{|l|}{ Armas } \\
\hline Sí & $25(10.6)$ & $16(12.2)$ & $9(6.9)$ & $4.309(1) *$ \\
\hline No & $211(89.4)$ & $89(84.8)$ & $122(93.1)$ & \\
\hline \multicolumn{5}{|l|}{ Agresor } \\
\hline Desconocido & $63(26.7)$ & $27(25.7)$ & $36(27.5)$ & $.760(1)$ n.s. \\
\hline Conocido & $173(73.3)$ & $78(74.3)$ & $95(72.5)$ & \\
\hline \multicolumn{5}{|l|}{ Denuncia } \\
\hline Sí & $115(48.7)$ & $52(49.5)$ & $63(48.1)$ & $.048(1)$ n.s. \\
\hline No & $121(51.3)$ & $53(50.5)$ & $68(51.9)$ & \\
\hline \multicolumn{5}{|c|}{ Victimización secundaria (115) } \\
\hline Sí & $32(27.8)$ & $15(288)$ & $17(27.0)$ & \\
\hline No & $83(72.2)$ & $37(71.2)$ & $46(73.0)$ & $.049(1)$ n.s. \\
\hline \multicolumn{5}{|l|}{$\mathrm{H}^{\mathrm{a}}$ de victimización } \\
\hline Sí & $55(23.3)$ & $22(20.9)$ & $33(25.2)$ & $.586(1) \mathrm{n} . \mathrm{s}$ \\
\hline No & $181(76.7)$ & $83(79.1)$ & $98(74.8)$ & \\
\hline \multicolumn{5}{|l|}{ Sucesos estresantes } \\
\hline Sí & $95(40.3)$ & $52(49.5)$ & $43(32.8)$ & $6.758(1) * *$ \\
\hline No & $141(59.7)$ & $53(50.5)$ & $88(67.2)$ & \\
\hline \multicolumn{5}{|l|}{ Abuso de drogas } \\
\hline Sí & $19(8)$ & $13(12.4)$ & $6(4.6)$ & $4.791(1) *$ \\
\hline No & $217(92)$ & $92(87.6)$ & $125(95.4)$ & \\
\hline \multicolumn{5}{|l|}{ Apoyo social } \\
\hline Sí & $166(70.3)$ & $64(60.9)$ & $102(77.9)$ & $7.988(1) * *$ \\
\hline No & $70(29.7)$ & $41(39.1)$ & $29(22.1)$ & \\
\hline \multicolumn{5}{|l|}{ Contar lo ocurrido } \\
\hline Sí & $198(83.9)$ & $89(84.8)$ & $109(83.2)$ & $.104(1)$ n.s. \\
\hline No & $38(16.1)$ & $16(15.2)$ & $22(16.8)$ & \\
\hline \multicolumn{5}{|l|}{ Apoyo familiar (198) } \\
\hline Sí & $119(60.1)$ & $50(56.8)$ & $69(63.3)$ & $1.037(1)$ n.s. \\
\hline No & $79(39.9)$ & $39(43.8)$ & $40(36.7)$ & \\
\hline \multicolumn{5}{|l|}{ Apoyo de pareja (90) } \\
\hline Sí & $72(80)$ & $26(78.8)$ & $46(80.7)$ & \\
\hline No & $18(20)$ & $7(21.2)$ & $11(19.3)$ & $.048(1)$ n.s. \\
\hline \multicolumn{5}{|l|}{ Amenaza a la vida } \\
\hline Sí & $68(28.8)$ & $36(34.3)$ & $32(24.4)$ & 2.762(1) n.s. \\
\hline No & $168(71.2)$ & $69(65.7)$ & $99(75.6)$ & \\
\hline
\end{tabular}

$* p<.05 . * * p<.01 . * * * p<.001$ 
Tabla 3. Relación entre las características de la agresión sexual y las variables relacionadas con la víctima y el apoyo recibido en relación con el malestar emocional (ME)

\begin{tabular}{|c|c|c|c|c|}
\hline & $\begin{array}{c}\text { Muestra }(\mathrm{N}=236) \\
\mathrm{N}(\%)\end{array}$ & $\begin{array}{c}\text { Con ME }(\mathrm{N}=150) \\
\mathrm{N}(\%)\end{array}$ & $\begin{array}{c}\text { Sin ME }(N=86) \\
N(\%)\end{array}$ & $\chi^{2}(\mathrm{gl})$ \\
\hline \multicolumn{5}{|l|}{ Tipo de agresión } \\
\hline Con penetración & $108(45.8)$ & $72(48.0)$ & $36(41.9)$ & \multirow[t]{2}{*}{$.830(1)$ n.s. } \\
\hline Sin penetración & $128(54.2)$ & $78(52.0)$ & $50(58.1)$ & \\
\hline \multicolumn{5}{|l|}{ Edad del abuso } \\
\hline Infancia & $121(51.3)$ & $81(54.0)$ & $40(46.5)$ & \multirow[t]{2}{*}{$1.227(1)$ n.s. } \\
\hline Edad adulta & $115(48.7)$ & $69(46.0)$ & $46(53.5)$ & \\
\hline \multicolumn{5}{|l|}{ Frecuencia } \\
\hline Ocasional & $114(48.3)$ & $64(42.7)$ & $50(58.1)$ & \multirow[t]{2}{*}{$5.241(1) *$} \\
\hline Repetida & $122(51.7)$ & $86(57.3)$ & $36(41.9)$ & \\
\hline \multicolumn{5}{|l|}{ Lesiones } \\
\hline Sí & $41(17.4)$ & $26(17.3)$ & $15(17.4)$ & \multirow[t]{2}{*}{$.000(1)$ n.s. } \\
\hline No & $195(82.6)$ & $124(82.7)$ & $71(82.6)$ & \\
\hline \multicolumn{5}{|l|}{ Armas } \\
\hline Sí & $25(10.6)$ & $14(10.3)$ & $11(12.8)$ & \multirow[t]{2}{*}{ 690(1) n.s. } \\
\hline No & $211(89.4)$ & $136(90.7)$ & $75(87.2)$ & \\
\hline \multicolumn{5}{|l|}{ Agresor } \\
\hline Desconocido & $63(26.7)$ & $34(22.7)$ & $29(33.7)$ & \multirow[t]{2}{*}{$3.613(1) *$} \\
\hline Conocido & $173(73.3)$ & $116(77.3)$ & $57(66.2)$ & \\
\hline \multicolumn{5}{|l|}{ Denuncia } \\
\hline Sí & $115(48.7)$ & $69(46.0)$ & $46(53.5)$ & \multirow[t]{2}{*}{ 1.227(1) n.s. } \\
\hline No & $121(51.3)$ & $81(54.0)$ & $40(46.5)$ & \\
\hline \multicolumn{5}{|c|}{ Victimización secundaria (115) } \\
\hline Sí & $32(27.8)$ & $25(36.2)$ & $7(15.2)$ & \multirow[t]{2}{*}{$6.069(1) *$} \\
\hline No & $83(72.2)$ & $44(63.8)$ & $39(84.8)$ & \\
\hline \multicolumn{5}{|l|}{$\mathrm{H}^{\mathrm{a}}$ de victimización } \\
\hline Sí & $55(23.3)$ & $38(25.3)$ & $17(19.8)$ & \multirow[t]{2}{*}{$.947(1)$ n.s. } \\
\hline No & $181(76.7)$ & $112(74.7)$ & $69(80.2)$ & \\
\hline \multicolumn{5}{|l|}{ Sucesos estresantes } \\
\hline Sí & $95(40.2)$ & $75(50.0)$ & $20(23.2)$ & \multirow[t]{2}{*}{$16.256(1)$ *** } \\
\hline No & $141(59.8)$ & $75(50.0)$ & $66(76.7)$ & \\
\hline \multicolumn{5}{|l|}{ Abuso de drogas } \\
\hline Sí & $19(8)$ & $16(10.7)$ & $3(3.5)$ & \multirow[t]{2}{*}{$3.805(1) *$} \\
\hline No & $217(92)$ & $134(89.3)$ & $83(96.5)$ & \\
\hline Apoyo social & & & & \\
\hline Sí & $166(70.3)$ & $93(62.0)$ & $73(84.9)$ & $13.720(1) * * *$ \\
\hline No & $70(29.7)$ & $57(38.0)$ & $13(15.1)$ & \\
\hline Contar lo ocurrido & & & & \\
\hline Sí & $198(83.9)$ & $127(84.7)$ & $71(82.6)$ & $.180(1)$ n.s. \\
\hline No & $38(16.1)$ & $23(15.3)$ & $15(17.4)$ & \\
\hline Apoyo familiar (198) & & & & \\
\hline Sí & $119(60.1)$ & $68(53.5)$ & $51(71.8)$ & $6.351(1) *$ \\
\hline No & $79(39.9)$ & $59(46.5)$ & $20(28.2)$ & \\
\hline Apoyo de pareja (90) & & & & \\
\hline Sí & $72(80)$ & $39(72.2)$ & $33(91.7)$ & $5.104(1) *$ \\
\hline No & $18(20)$ & $15(27.8)$ & $3(8.3)$ & \\
\hline Amenaza a la vida & & & & \\
\hline Sí & $68(28.8)$ & $44(29.3)$ & $24(27.9)$ & $.054(1)$ n.s. \\
\hline No & $168(71.2)$ & $106(70.7)$ & $62(72.1)$ & \\
\hline
\end{tabular}

${ }^{*} p<.05 . * * p<.01 . * * * p<.001$ 
la exposición a una historia de victimización anterior (el $28.9 \%$ ) y a sucesos especialmente estresantes en el último año (el 54.4\%), así como la falta de apoyo social (el 44.7\%) y familiar (el $47.9 \%$ ), estuvieron presentes en mayor proporción en las víctimas con sentimientos de culpa (tabla 4).

Además, las víctimas con sentimientos de culpa tardaron más tiempo en ponerse en contacto con el programa $(X=10.6$ años; $D T=10.8$ años) en comparación con las que no los tenían ( $X=5.7$ años; $D T=8.5$ años) $[t(234)=3.871 ; p<.001]$.

\section{d) Características de la agresión sexual y variables relacionadas con la víctima y con el apoyo recibido en relación con un déficit de autoestima}

Las víctimas con baja autoestima sufrieron con mayor frecuencia una agresión con penetración vaginal o anal (el 51.1\%), repetida (el 60.1\%), experimentada durante su infancia (el 56.7\%) y perpetrada por un conocido (el $79.0 \%$ ), en comparación con las que tenían una autoestima satisfactoria. Asimismo, la exposición a una historia de victimización anterior (el 28.7\%), a sucesos especialmente estresantes en el último año (el 53.2\%), así como la falta de apoyo social (el $37.8 \%$ ), familiar (el $47.9 \%$ ) y de pareja (el $29.1 \%$ ), aparecieron en mayor proporción en las víctimas con un déficit de autoestima (tabla 5).

Además, las víctimas con una baja autoestima tardaron más tiempo en ponerse en contacto con el programa $(X=9.3$ años; $D T=10.4$ años) en comparación con las que tenían una autoestima satisfactoria ( $X=6.3$ años; $D T=9.1$ años) $[t$ $(234)=2.272 ; p<.05]$.

Tabla 4. Relación entre las características de la agresión sexual y las variables relacionadas con la víctima y el apoyo recibido en relación con los sentimientos de culpa (SC)

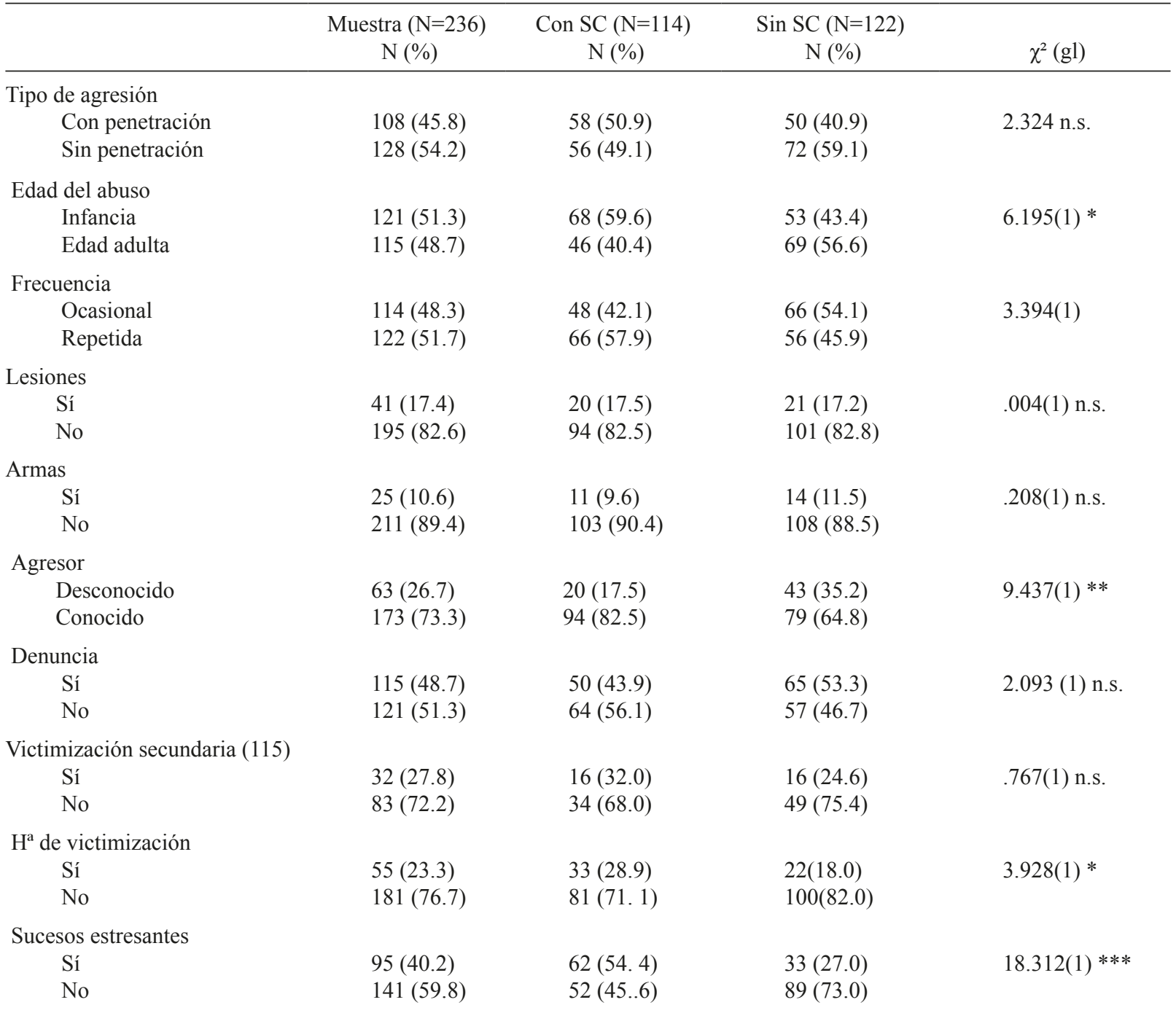


Continuación Tabla 4

\begin{tabular}{|c|c|c|c|c|}
\hline & $\begin{array}{c}\text { Muestra }(\mathrm{N}=236) \\
\mathrm{N}(\%)\end{array}$ & $\begin{array}{c}\text { Con SC }(\mathrm{N}=114) \\
\mathrm{N}(\%)\end{array}$ & $\begin{array}{c}\text { Sin } \mathrm{SC}(\mathrm{N}=122) \\
\mathrm{N}(\%)\end{array}$ & $\chi^{2}(\mathrm{gl})$ \\
\hline \multicolumn{5}{|c|}{ Abuso de drogas } \\
\hline Sí & $19(8.1)$ & $13(11.4)$ & $6(4.9)$ & $3.348(1)+$ \\
\hline No & 217 (91.9) & $101(88.6)$ & $116(95.1)$ & \\
\hline \multicolumn{5}{|c|}{ Apoyo social } \\
\hline Sí & $166(70.3)$ & $63(55.3)$ & $103(84.4)$ & $24.024(1) * * *$ \\
\hline No & $70(29.7)$ & $51(44.7)$ & $19(15.6)$ & \\
\hline \multicolumn{5}{|c|}{ Contar lo ocurrido } \\
\hline Sí & $198(83.9)$ & $94(82.4)$ & $104(85.2)$ & $.340(1)$ n.s. \\
\hline No & $38(16.1)$ & $20(17.5)$ & $18(14.8)$ & \\
\hline \multicolumn{5}{|c|}{ Apoyo familiar (198) } \\
\hline Sí & $119(60.1)$ & $45(47.9)$ & $74(71.1)$ & $11.160(1) * *$ \\
\hline No & $79(39.9)$ & $49(52.1)$ & $30(28.9)$ & \\
\hline \multicolumn{5}{|c|}{ Apoyo de pareja (90) } \\
\hline Sí & $72(80.0)$ & $31(73.8)$ & $41(85.4)$ & 1.886(1) n.s. \\
\hline No & $18(20.0)$ & $11(26.2)$ & $7(14.6)$ & \\
\hline \multicolumn{5}{|c|}{ Amenaza a la vida } \\
\hline Sí & $68(28.8)$ & $29(25.4)$ & $39(32.0)$ & $1.225(1)$ n.s. \\
\hline No & $168(71.2)$ & $85(74.6)$ & $83(68.0)$ & \\
\hline
\end{tabular}

$* p<.05 . * * p<.01 . * * * p<.001$

Tabla 5. Relación entre las características de la agresión sexual y las variables relacionadas con la víctima y el apoyo recibido en relación con la baja autoestima (BAU)

\begin{tabular}{|c|c|c|c|c|}
\hline & Muestra $(\mathrm{N}=236)-\mathrm{N}(\%)$ & Con BAU $(\mathrm{N}=143)-\mathrm{N}(\%)$ & Sin BAU $(N=93)-N(\%)$ & $\chi^{2}(\mathrm{gl})$ \\
\hline \multicolumn{5}{|l|}{ Tipo de agresión } \\
\hline Con penetración & $108(45.8)$ & $73(51.1)$ & $35(37.6)$ & \multirow[t]{2}{*}{$4.086(1) *$} \\
\hline Sin penetración & $128(54.2)$ & $70(48.9)$ & $58(62.6)$ & \\
\hline \multicolumn{5}{|l|}{ Edad del abuso } \\
\hline Infancia & $121(51.3)$ & $81(56,7)$ & $40(43,0)$ & \multirow[t]{2}{*}{$4.192(1) *$} \\
\hline Edad adulta & $115(48.7)$ & $62(43,3)$ & $53(56,0)$ & \\
\hline \multicolumn{5}{|l|}{ Frecuencia } \\
\hline Ocasional & $114(48,3)$ & $57(39,9)$ & $57(51,3)$ & \multirow[t]{2}{*}{$10.364(1) * *$} \\
\hline Repetida & $122(51,7)$ & $86(60,1)$ & $36(38.8)$ & \\
\hline \multicolumn{5}{|l|}{ Lesiones } \\
\hline Sí & $41(17,4)$ & $24(16,8)$ & $17(18,3)$ & \multirow[t]{2}{*}{ 0,088(1) n.s. } \\
\hline No & $195(82,6)$ & $119(83,2)$ & $76(81,7)$ & \\
\hline \multicolumn{5}{|l|}{ Armas } \\
\hline Sí & $25(10,6)$ & $15(10,5)$ & $10(10,7)$ & \multirow[t]{2}{*}{ 0.004(1) n.s. } \\
\hline No & $211(89,4)$ & $128(89,5)$ & $83(89.3)$ & \\
\hline \multicolumn{5}{|l|}{ Agresor } \\
\hline Desconocido & $63(26.7)$ & $30(21.0)$ & $33(35.5)$ & \multirow[t]{2}{*}{$6.059(1) *$} \\
\hline Conocido & $173(73.3)$ & $113(79.0)$ & $60(64.5)$ & \\
\hline \multicolumn{5}{|l|}{ Denuncia } \\
\hline Sí & $115(48.7)$ & $63(44.1)$ & $52(55.9)$ & \multirow[t]{2}{*}{$3.172(1)$} \\
\hline No & $121(51.3)$ & $80(55.9)$ & $41(44.1)$ & \\
\hline
\end{tabular}




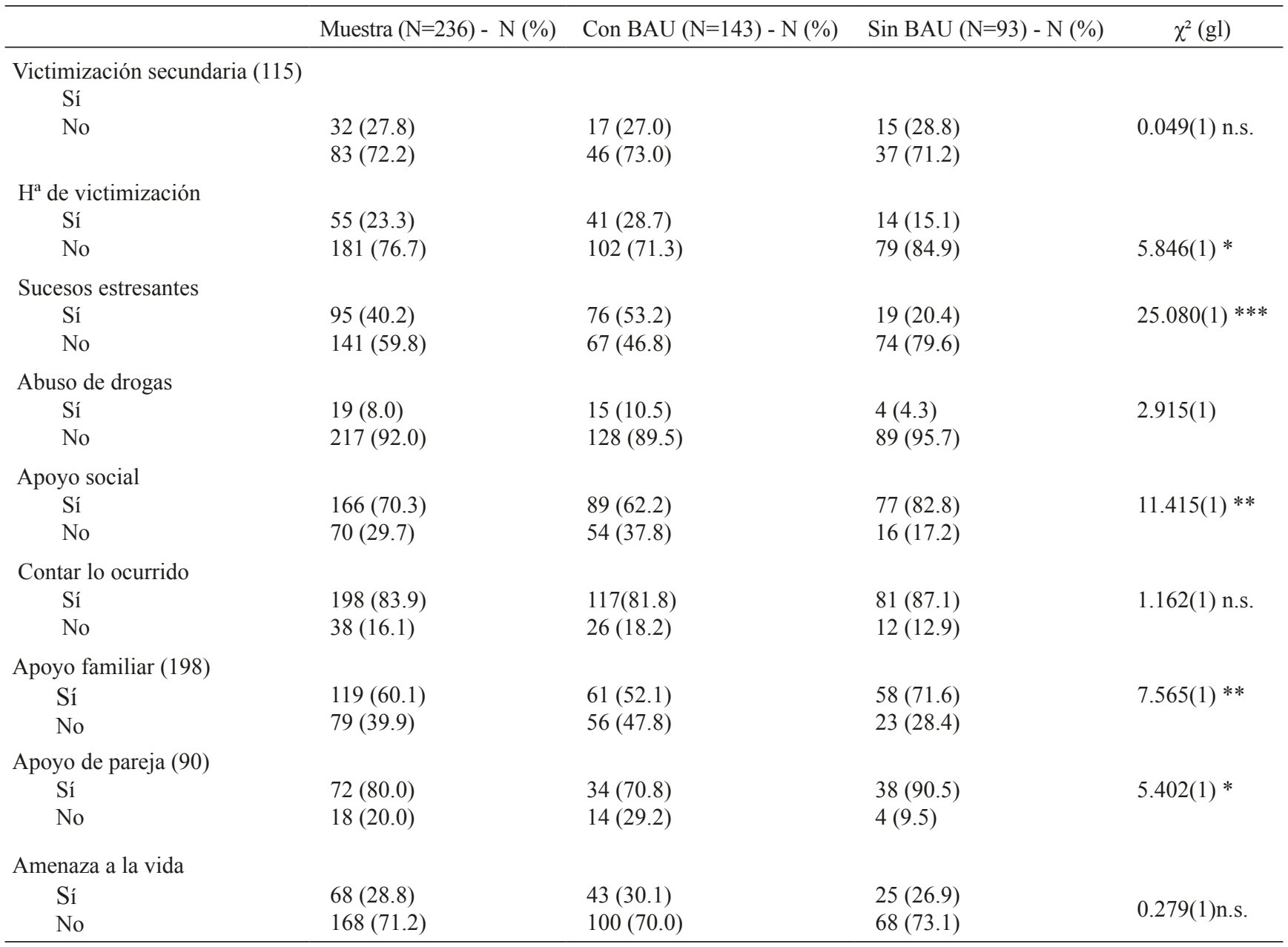

$* \mathrm{p}<.05 .{ }^{* *} \mathrm{p}<.01 . * * * \mathrm{p}<.001$

e) Características de la agresión sexual y variables relacionadas con la víctima y con el apoyo recibido en relación con la evitación sexual

La presencia o ausencia de problemas de evitación sexual resultó ser independiente de las características de la agresión, así como de las variables relacionadas con la víctima y con el apoyo recibido. Por ejemplo, el tipo de agresión (con o sin penetración) $\left[X^{2}(1)=.210 ;\right.$ n.s. $]$, la existencia de lesiones $\left[X^{2}\right.$ $(1)=3.532 ; p<.10]$, la relación con el agresor (desconocido o conocido) $\left[X^{2}(1)=.281\right.$; n.s. $]$, así como la exposición a sucesos especialmente estresantes $\left[X^{2}(1)=.629\right.$; n.s. $]$ o a una historia de victimización anterior $\left[X^{2}(1)=3.003 ; p<.10\right]$ o la presencia de apoyo social $\left[X^{2}(1)=1.145 ;\right.$ n.s. $]$ y familiar $\left[X^{2}\right.$ $(1)=.549 ;$ n.s. $]$, no mostraron diferencias estadísticamente significativas. Sin embargo, estos resultados conviene interpretarlos con cautela dado que muchas de las víctimas o no tenían pareja ( $n=146$, el $61.9 \%)$ o no presentaban este problema en la evaluación pretratamiento ( $n=35$, el $38.9 \%)$.

\section{Discusión}

En esta investigación se han estudiado los factores que aumentan el riesgo de un mayor impacto emocional en una muestra de 269 mujeres adultas víctimas de agresiones sexuales, en la infancia o en la vida adulta, en tratamiento. Se trata, por tanto, de una muestra amplia de mujeres jóvenes (en torno a 25-30 años) que presentaban un malestar emocional crónico que desbordaba sus recursos psicológicos de afrontamiento y que interfería negativamente en su vida cotidiana, tal y como se muestra también en otros trabajos (Cortés et al., 2011; Fergusson et al., 2008; Vickerman y Margolin, 2009).

Las víctimas estudiadas en esta investigación han sufrido una agresión sexual grave, con una penetración vaginal o anal y de forma repetida en casi la mitad de los casos. Los agresores sexuales eran en una gran mayoría conocidos de las víctimas. Los síntomas o trastornos clínicos pueden ser atribuidos a los efectos de la agresión sexual, porque en dos de cada tres casos no presentaban otra vía de victimización 
ni las víctimas eran consumidoras abusivas de alcohol o drogas (Sarasua et al., 2012, en prensa).

Las víctimas han denunciado la agresión sexual solo en la mitad de los casos, especialmente cuando el agresor es un desconocido y cuando la agresión ha tenido lugar recientemente en la vida adulta. La presentación de la denuncia está asociada a la gravedad de la conducta sexual (violación), a la existencia de lesiones y a la utilización de armas, lo que implica la percepción de amenaza a la vida. La denuncia constituye un elemento de protección porque se relaciona con un mayor apoyo familiar y social, según se indica también en investigaciones previas (Resick et al., 2012).

Por lo que se refiere a las características psicopatológicas de la muestra, las víctimas presentaban cuadros clínicos diversos porque la agresión sexual es un suceso traumático, no un diagnóstico clínico (Echeburúa et al., 2002; Maniglio, 2009). Los síntomas o trastornos más frecuentes en la muestra estudiada eran el malestar emocional (un 63.6\% del total), los déficits de autoestima (un 59.7\%), los sentimientos de culpa (un $48.3 \%$ ), el trastorno de estrés postraumático (un $44.5 \%$ ) y las conductas de evitación sexual (un 38.9\%), como también aparece en otros estudios (Campbell y Wasco, 2005; Foa y Rothbaum, 1998).

La gravedad del impacto emocional está relacionada directamente con las circunstancias de la agresión sexual. Así, la existencia de una penetración vaginal o anal, la presencia de lesiones o el recurso a un arma como factor de intimidación se asocian a un TEPT. El malestar emocional, sin embargo, está más vinculado a la frecuencia repetida de la agresión sexual y a la presencia de un agresor conocido de la víctima. Los sentimientos de culpa y los déficits de autoestima, a su vez, aparecen más cuando las víctimas han sufrido un abuso sexual reiterado en la infancia y el agresor es una persona cercana a ellas. Por último, las conductas de evitación sexual no están asociadas directamente a las circunstancias de la agresión sexual. Estos resultados son congruentes con los estudios de Echeburúa et al. (2012, en prensa) y de Sarasua et al. (2012, en prensa).

Hay circunstancias de victimización y otros factores psicosociales que también están asociados a la gravedad del impacto emocional y que son relativamente similares en los distintos síntomas o cuadros clínicos. De este modo, la existencia de sucesos estresantes en el último año, el consumo abusivo de alcohol o drogas y la ausencia de un apoyo social adecuado están relacionados directamente con el TEPT. El malestar emocional está más asociado a la victimización secundaria, a la existencia de sucesos estresantes, al consumo abusivo de alcohol o drogas y a la ausencia de apoyo generalizado (familiar, social y de pareja). Los sentimientos de culpa y los déficits de autoestima, a su vez, aparecen más cuando las víctimas presentan una historia de victimización, sufren sucesos estresantes el último año y carecen de apoyo familiar y social. Por último, las conductas de evitación sexual no están asociadas directamente a factores de victimización o a factores psicosociales.

El apoyo social en un sentido amplio (pareja, familia y relaciones sociales) constituye un elemento de protección y previene hasta cierto punto la aparición de una sintomatología psicopatológica en las víctimas de agresiones sexuales. Por el contrario, la victimización secundaria, cuando se presenta una denuncia y la reacción del sistema judicial es inadecuada, tiende a agravar el cuadro clínico en este tipo de víctimas, como ocurre también en el caso de las víctimas de violencia de género (Echeburúa, Fernández-Montalvo, Corral y López-Goñi, 2009; Sarasua, Zubizarreta, Echeburúa y Corral, 2007).

Otro dato de interés, que puede resultar paradójico, es que las víctimas con una mayor sintomatología (malestar emocional, sentimientos de culpa y déficits de autoestima) tardan más tiempo en buscar ayuda terapéutica. Ello plantea la necesidad de diseñar estrategias motivacionales para atraer a estas víctimas al tratamiento.

A diferencia de otros estudios previos centrados exclusivamente en el TEPT (cfr. Foa y Rothbaum, 1998; Taylor y Harvey, 2009), los resultados expuestos en este estudio están referidos a un espectro de conductas más amplio (TEPT, malestar emocional, evitación sexual, sentimientos de culpa y déficits de autoestima), que se corresponde mejor con la demanda terapéutica en las víctimas de agresiones sexuales. Otra ventaja de este estudio es que permite predecir un mapa, todavía incompleto, de las reacciones psicopatológicas en función de las circunstancias de la agresión sexual, de la victimización sufrida y de ciertos factores psicosociales, tales como la existencia reciente de factores estresantes o de abuso de alcohol/drogas y el grado de apoyo social/ familiar recibido. Todo ello permite diseñar un tratamiento más a la medida de la víctima.

Algunas limitaciones de esta investigación son que las víctimas estudiadas han acudido a un programa de tratamiento y que, a efectos de homogeneizar la muestra, se han excluido las mujeres agredidas sexualmente en el marco de la relación de pareja y las que han sufrido un acoso sexual en el ámbito laboral. Por tanto, las conclusiones obtenidas no se pueden generalizar a todas las víctimas que han estado expuestas a este tipo de sucesos. La investigación futura deberá prestar atención a estos puntos.

\section{Referencias}

Beck, A.T., Rush, A.J., Shaw, B.F., y Emery, G. (1979). Cognitive therapy of depression. New York: Guilford Press.

Beck, A.T., Brown, G.K., y Steer, R.A. (1996). Beck Depression Inventory (BDI-II). San Antonio, TX: The Psychological Corporation.

Briere, J., y Elliott, D.M. (2003). Prevalence and psychological sequelae of self-reported childhood physical and sexual abuse in a general population sample of men and women. Child Abuse and Neglect, 27, 1205-1222. 
Campbell, R., y Wasco, S.M. (2005). Understanding rape and sexual assault. Journal of Interpersonal Violence, 20, 127-131.

Cortés, M.R., Cantón-Cortés, D., y Cantón, J. (2011). Consecuencias a largo plazo del abuso sexual infantil: papel de la naturaleza y continuidad del abuso y del ambiente familiar. Behavioral Psychology/Psicología Conductual, 19, 41-56.

Echeburúa, E. (2010). The challenge of posttraumatic stress disorder prevention: how to survive a disaster? Terapia Psicológica, 28, 147-154.

Echeburúa, E., y Corral, P. (1998). Manual de violencia familiar. Madrid: Siglo XXI.

Echeburúa, E., y Corral, P. (2007). Intervención en crisis en víctimas de sucesos traumáticos: ¿cuándo, cómo y para qué? Psicología Conductual, 15, 373-387.

Echeburúa, E., y Guerricaechevarría, C. (2011). Tratamiento psicológico de las víctimas de abuso sexual infantil intrafamiliar: un enfoque integrador. Behavioral Psychology/Psicología Conductual, 19, 469-486.

Echeburúa, E., Corral, P., y Amor, P.J. (2002). Evaluación del daño psicológico en las víctimas de delitos violentos. Psicothema, 14 (supl.), 139-146.

Echeburúa, E., Corral, P., y Fernández-Montalvo, J. (2000). Escala de Inadaptación: propiedades psicométricas en contextos clínicos. Análisis y Modificación de Conducta, 26, 325-340.

Echeburúa, E., Corral, P., Sarasua, B., y Zubizarreta, I. (1996). Treatment of acute posttraumatic stress disorder in rape victims: An experimental study. Journal of Anxiety Disorders, 10, 185-199.

Echeburúa, E., Corral, P., Zubizarreta, I., y Sarasua, B. (1995). Trastorno de estrés postraumático crónico en víctimas de agresiones sexuales. La Coruña: Fundación Paideia.

Echeburúa, E., Corral, P., Zubizarreta, I., y Sarasua, B. (1997). Psychological treatment of chronic posttraumatic stress disorder in victims of sexual aggression. Behavior Modification, 21, 433-456.

Echeburúa, E., Corral, P., Amor, P.J., Zubizarreta, I., y Sarasua, B. (1997). Escala de gravedad de síntomas del trastorno de estrés postraumático: Propiedades psicométricas. Análisis y Modificación de Conducta, 23, 503-526.

Echeburúa, E., Fernández-Montalvo, J., Corral, P., y López-Goñi, J.J. (2009). Assessing the risk markers in femicide and in severe intimate partner violence: A new assessment instrument. Journal of Interpersonal Violence, 24, 925-939.

Echeburúa, E., Sarasua, B., Zubizarreta, I., y Corral, P. (2012, en prensa). Tratamiento psicológico de mujeres víctimas de agresiones sexuales recientes y no recientes en la vida adulta: un análisis comparativo. Behavioral Psychology/Psicología Conductual.
Fergusson, D.M., Boden, J.M., y Horwood, L.J. (2008). Exposure to childhood sexual and physical abuse and adjustment in early adulthood. Child Abuse and Neglect, 32, 607-619.

Foa, E.B., y Rothbaum, B.O. (1998). Treating the trauma of rape: Cognitive-behavioral therapy for PTSD. New York: Guilford Press.

Foa, E.B., Zoellner, L.A., y Feeny, N.C. (2006). An evaluation of three brief programs for facilitating recovery after assault. Journal of Traumatic Stress, 19, 29-43.

Maniglio, R. (2009). Severe mental illness and criminal victimization: A systematic review. Acta Psychiatrica Scandinavica, 119, 180-191.

Pereda, N., Guilera, G., Forns, M., y Gómez-Benito, J. (2009). The prevalence of child sexual abuse in community and student samples: A meta-analysis. Clinical Psychology Review, 29, 328-338.

Picó-Alfonso, M.A., Echeburúa, E., y Martínez, M. (2008). Personality disorder symptoms in women as a result of chronic intimate male partner violence. Journal of Family Violence, 23, 577-588.

Resick, P.A., Williams, L.F., Suvak, M.K., Monson, C.M., y Gradus, J.L. (2012). Long-term outcomes of cognitive-behavioral treatments for PTSD among female rape survivors. Journal of Consulting and Clinical Psychology, 80, 201-210.

Resnick, H., Acierno, R., Waldrop, A.E., King, L., King, D., Danielson, C., Ruggiero, K.J., y Kilpatrick, D.G. (2007). Randomized controlled evaluation of an early intervention to prevent post-rape psychopathology. Behaviour Research and Therapy, 45, 2342-2447.

Rosenberg, M. (1965). Society and the Adolescent Self Image. Princeton: Princeton University Press.

Sarasua, B., Zubizarreta, I., Echeburúa, E., y Corral, P. (2007). Perfil psicopatológico diferencial de las víctimas de violencia de pareja en función de la edad: un estudio exploratorio. Psicothema, 19, 459-466.

Sarasua, B., Zubizarreta, I., Corral, P., y Echeburúa, E. (2012, en prensa). Tratamiento psicológico de mujeres adultas víctimas de abuso sexual en la infancia: resultados a largo plazo. Anales de Psicología.

Spielberger, C.D., Gorsuch, R.L., y Lushene, R.E. (1970). Manual of State/ Trait Anxiety Inventory. Palo Alto CA: Consulting Psychologists Press (versión española, TEA, 1982).

Taylor, J.A., y Harvey, S.T. (2009). Effects of psychotherapy with people who have been sexually assaulted: A meta-analysis. Aggression and Violent Behavior, 14, 273-285.

Vázquez, C., y Sanz, J. (1997). Fiabilidad y valores normativos de la versión española del Inventario para la Depresión de Beck de 1978/ Reliability and norm data of the Spanish versión of the 1978 Beck Depression Inventory. Clínica y Salud, 8, 403-422.

Vickerman, K.A., y Margolin, G. (2009). Rape treatment outcome research: Empirical findings and state of the literature. Clinical Psychology Review, 29, 431-448. 